\title{
Fast and Efficient Dynamic WDM Semiconductor Optical Amplifier Model
}

\author{
Walid Mathlouthi, Pascal Lemieux, Massimiliano Salsi, Armando Vannucci, Member, IEEE, \\ Alberto Bononi, and Leslie A. Rusch, Senior Member, IEEE
}

\begin{abstract}
A novel state-variable model for semiconductor optical amplifiers (SOAs) that is amenable to block diagram implementation of wavelength division multiplexed (WDM) signals and fast execution times is presented. The novel model is called the reservoir model, in analogy with similar blockoriented models for Raman and erbium-doped fiber amplifiers (EDFAs). A procedure is proposed to extract the needed reservoir model parameters from the parameters of a detailed and accurate space-resolved SOA model due to Connelly, which was extended to cope with the time-resolved gain transient analysis. Several variations of the reservoir model are considered with increasing complexity, which allow the accurate inclusion of scattering losses and gain saturation induced by amplified spontaneous emission. It is shown that at comparable accuracy, the reservoir model can be $\mathbf{2 0}$ times faster than the Connelly model in single-channel operation; much more significant time savings are expected for WDM operation. The model neglects intraband SOA phenomena and is thus limited to modulation rates per channel not exceeding $10 \mathrm{~Gb} / \mathrm{s}$. The SOA reservoir model provides a unique tool with reasonably short computation times for a reliable analysis of gain transients in WDM optical networks with complex topologies.
\end{abstract}

Index Terms-Optical pulse amplification, semiconductor optical amplifier (SOA).

\section{INTRODUCTION}

$\mathbf{S}$ EMICONDUCTOR optical amplifiers (SOAs) are becoming key devices for future optical networks. SOAs are used in a wealth of applications to achieve highly varied functions. For example, optical switching and wavelength conversion can be accomplished using cross-gain modulation (XGM), four-wave mixing (FWM), or cross-phase modulation (XPM) [1]-[3]. Signal reshaping and noise cleaning of ON-OFF keying (OOK) signals are also feasible using saturated SOAs [4], with particularly effective application in spectrum sliced wavelength-division multiplexing (WDM) [5] and incoherent optical code-division multiple access [6].

Manuscript received March 15, 2006; revised July 18, 2006. This work was supported in part by FQRNT under Grant PADCO FT076892, the Canadian Institute for Photonic Innovation under Grant CG082485, and the Italian Ministry under Project WONDER.

W. Mathlouthi, P. Lemieux, and L. A. Rusch are with the Center of Optics, Photonics and Lasers, Département de Génie Électrique et Informatique, Université Laval, Ste-Foy, QC G1K 7P4, Canada (e-mail: mathlout@gel.ulaval.ca; plemieux@gel.ulaval.ca; rusch@gel.ulaval.ca).

M. Salsi, A. Vannucci, and A. Bononi are with the Dipartimento di Ingegneria dell'Informazione, Universitá di Parma, 43100 Parma, Italy (e-mail: salsi@ tlc.unipr.it; bononi@tlc.unipr.it).

Color versions of Figs. 2-4, 6-9, and 11-16 are available online at http://ieeexplore.ieee.org.

Digital Object Identifier 10.1109/JLT.2006.884217
In this paper, we are interested in analyzing the response of SOAs to optical signals that are modulated at bit rates not exceeding $10 \mathrm{~Gb} / \mathrm{s}$, such as those planned for next-generation metropolitan area networks. Therefore, ultrafast intraband phenomena such as carrier heating $(\mathrm{CH})$ and spectral hole burning (SHB) (see, e.g., [7]-[9]) can be neglected, and only carrierinduced gain dynamics need to be included, as was done in several SOA models developed in the past. Such models can be divided into two broad categories: 1) space-resolved numerically intensive models, which take into account facet reflectivity as well as forward and backward propagating signals and amplified spontaneous emission (ASE) and offer a good fit to experimental data [10]-[12], and 2) simplified analytical models with a coarser fit to experimental data but developed to facilitate conceptual understanding and performance analysis [2], [13]-[16]. For the purpose of carrying out extensive Monte Carlo simulations for statistical signal analysis and bit-errorrate (BER) estimation, the accurate space-resolved models are ruled out because of their prohibitively long simulation times. However, a simplified model with a satisfactory fit to experimental results would be highly desirable. Most simplified models can be derived from the work of Agrawal and Olsson [13]. Under suitable assumptions, Agrawal and Olsson managed to reduce the coupled propagation and rate equations into a single ordinary differential equation (ODE) for the integrated gain [13, eq. (3.4)]. The simplicity of the solution is due to the fact that waveguide scattering losses and ASE were neglected. ASE has an important effect on the spatial distribution of carrier density and saturation, and it may significantly affect the SOA steady-state and dynamic responses [17], [18]. Scattering losses also have an impact on the dynamic response of the SOA [3]. Moreover, Agrawal and Olsson's model was originally cast for single-wavelength-channel amplification, although it can be extended to multiwavelength operation by assuming that the channels are spaced far enough apart to neglect FWM beating in the copropagating case [3]. Saleh [14] arrived independently at the same model as Agrawal and Olsson's ([14, eq. (4a)] coincides with [13, eq. (3.4)]) and then introduced further simplifying approximations to get to a very simple block diagram of the single-channel SOA, which was exploited for a mathematically elegant stochastic performance analysis of single-channel saturated SOAs [15]. The loss of accuracy due to Saleh's extra approximations with respect to Agrawal's model was quantified in [19]. Saleh's model was later extended to cope with injection current modulation, scattering losses, and ASE [16]. In addition, Agrawal's model was extended to include ASE [20]. In both [16] and [20], ASE was added phenomenologically at the 


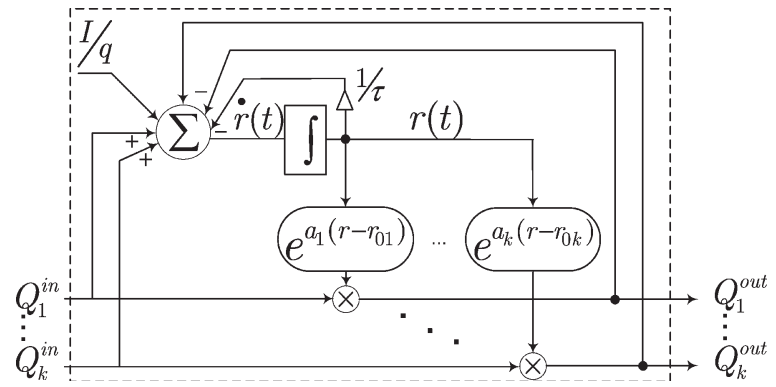

Fig. 1. Block diagram of the reservoir model. ASE contribution not shown for ease of drawing.

output of the SOA and did not influence the gain dynamics, thereby limiting the application to very small saturation levels.

In this paper, we first develop a dynamic version of the steady-state wideband SOA Connelly model [12], which is shown to fit quite well with our dynamic SOA experiments with OOK channels. The Connelly model was selected because it derives the SOA material gain coefficient from quantum mechanical principles without the assumption of linear dependence on carrier density that was made in [10] and [11]. Our dynamic Connelly model serves then as a benchmark to test the accuracy and computational-speed improvement of a novel state-variable SOA dynamic model, which represents the most important contribution of this paper. The novel model is an extension of Agrawal's model, as provided in [3], with the inclusion of approximations for scattering loss and ASE to better fit the experimental results and the dynamic Connelly model predictions. In such a model, the SOA dynamic behavior is reduced to the solution of a single ODE for the single state variable of the system, which is proportional to the integrated carrier density [3], which, for WDM operation, is a more appropriate variable than the integrated gain used in [13]. Once the state-variable dynamic behavior is found, the behavior of all the output WDM channels is also obtained. The state variable is called "reservoir" since it plays the same role as the reservoir of excited erbium ions in an erbium-doped fiber amplifier (EDFA) [21], [22]. Quite interestingly, then, the SOA for WDM operation admits almost the same block diagram description as that of an EDFA suggested by [21, eq. (5)]. Such a novel SOA block diagram is shown in Fig. 1 (without ASE for ease of drawing) and will be derived in the next sections. Note that this model treats the intensity of the electrical field, but the field phase can be indirectly obtained since it is a deterministic function of the reservoir [13]. In the SOA, the role of the optical pump for EDFAs is played by the injected current $I$. The most striking difference between the two kinds of amplifiers is the fluorescence time $\tau$, which is of the order of milliseconds in EDFAs and of a fraction of nanosecond in SOAs. Such a huge difference accounts for most of the disparity in the dynamic behavior between the two kinds of amplifiers and explains why SOAs have not been used for WDM applications for a long time [23]. However, recent cheap gain-clamped SOAs [24] are likely to promote the use of SOAs for WDM metro applications. As already mentioned, the reservoir model requires the (copropagating) WDM channels to have minimum channel spacing in excess of a few tens of gigahertz, in order to neglect the carrier-induced FWM fields generated in the SOA. This should not be a problem for channels allocated on the International Telecommunications Union grid with $50 \mathrm{GHz}$ spacing or more. However, an intrinsic limit of the reservoir model is its neglecting SHB and $\mathrm{CH}$, which generate FWM and XPM interactions among WDM channels even when the minimum channel spacing is large enough to rule out any carrier-induced interaction [9]. The predictions of the reservoir model will be accurate whenever the carrierinduced XGM mechanism dominates over FWM and XPM. It is worth mentioning that state-variable amplifier block diagrams are very important simulation tools that enable the reliable power propagation of WDM signals in optical networks with complex topologies; therefore, the present reservoir SOA model provides a new entry aside from the already known models for EDFAs [21], [25] and for Raman amplifiers [26]. A challenge in our reservoir model, as in all simplified SOA models, is to correctly choose the values of the wavelength-dependent coefficients that give the best fit to the experimental results. We propose and describe here a methodology to extract the needed wavelength-dependent coefficients from the parameters of the dynamic Connelly model.

This paper is organized as follows. In Section II, the dynamic Connelly model is introduced, and a procedure to derive its parameters from experiments is described. In Section III, the SOA reservoir model is derived first without ASE and then with ASE that is resolved over a large number of wavelength bins. Simulations show good accordance between the reservoir model predictions and experiments, and good improvement in calculation time with respect to the Connelly model. However, inclusion of many ASE wavelength channels makes even the reservoir model too slow for the BER estimations we have in mind. Hence, in order to further simplify the model, we introduce the reservoir model with a single equivalent ASE channel. The ASE can be seen as an independent input-signal channel (with proper input power and wavelength) that depletes the reservoir of a noiseless SOA. Results show that this last model is the most efficient one since it can be made to accurately predict experimental results with an execution time that is 20 times faster than that of the dynamic Connelly model for single-channel operation, with the savings increasing with the number of WDM signal channels. In Section III-C, we examine a model that was obtained by dividing the SOA into several sections, each characterized by its own reservoir. Here again, the ASE can be modeled as a single channel that propagates through the different reservoir stages. Results show better precision, although the increase in precision is not worth, in most cases, the loss in execution time. Most of the numerical results are reported in Section IV. Finally, Section V summarizes the main findings of this paper.

\section{DynAmic CONNElly Model}

\section{A. Theory}

In this paper, we adopt the wideband model for a bulk SOA proposed in [12], which is based on the numerical solution of the coupled equations for carrier-density rate and photonflux propagation for both the forward and backward signals 
and the spectral components of ASE. At a specified time $t$ and position $z$ in the SOA, the propagation equation of photon flux $Q_{k}^{ \pm}$[photons/s] of the $k$ th forward (+) or backward (-) signal is

$$
\frac{d Q_{k}^{ \pm}(z, t)}{d z}=\left\{ \pm\left[\Gamma g_{k}(N)-\alpha(N)\right]\right\} Q_{k}^{ \pm}(z, t)
$$

where $\Gamma$ is the fundamental mode confinement factor, $g_{k}$ is the material gain coefficient at the optical frequency $\nu_{k}$ of the $k$ th signal, $\alpha$ is the material-loss coefficient, and both are functions of carrier density $N(z, t)$. The power of the propagating signal is related to its photon flux as $P_{k}^{ \pm}=h \nu_{k} Q_{k}^{ \pm}$(in watts), where $h$ is Planck's constant. The ASE photon flux on each ASE wavelength channel obeys a similar propagation equation given by

$$
\frac{d Q_{j}^{ \pm}(z, t)}{d z}= \pm\left[\Gamma g_{j}(N)-\alpha(N)\right] Q_{j}^{ \pm}(z, t)+R_{\mathrm{sp}, j}(N)
$$

where $R_{\mathrm{sp}, j}(N)$ is the spontaneous emission rate coupled into the ASE channel at frequency $\nu_{j}$. The expression of $R_{\mathrm{sp}, j}(N)$ is given in [12, eq. (40)]. This expression will be used in Section III-B to develop a reservoir model equation that takes ASE into account. The carrier density at coordinate $z$ evolves as [12]

$$
\begin{aligned}
\frac{d N(z, t)}{d t}= & \frac{I}{q d L W}-R(N(z, t)) \\
& -\frac{\Gamma}{d W}\left\{\sum_{k=1}^{n_{\mathrm{sig}}} g_{k}(N)\left(Q_{k}^{+}(z, t)+Q_{k}^{-}(z, t)\right)\right\} \\
& -\frac{2 \Gamma}{d W}\left\{\sum_{j=1}^{n_{\mathrm{ASE}}} g_{j}(N) K_{j}\left(Q_{j}^{+}(z, t)+Q_{j}^{-}(z, t)\right)\right\}
\end{aligned}
$$

where $I$ is the bias current; $q$ is the electron charge; $d, L$, and $W$ are the active-region thickness, length, and width, respectively, and $R(N)$ is the recombination rate. An expression for $R(N)$ is given in [12, eq. (49)]; the reservoir model of Section III uses a linear approximation for $R(N)$ in (9); $n_{\mathrm{sig}}$ is the number of WDM signals; $n_{\mathrm{ASE}}$ is the number of spectral components of the ASE; and $K_{j}$ is an ASE multiplying factor, which equals 1 for zero facet reflectivity [12]. The factor 2 in (3) accounts for two ASE polarizations. Note that (3) contains an important approximation: it is the sum of the signals and ASE powers (fluxes), instead of-more correctly-the power of the sum of the signals and ASE fields, that depletes carrier density $N$. Therefore, (3) neglects the carrier-density pulsations due to beating among WDM channels that generate FWM and XPM in SOAs [9]. Although such an approximation is inappropriate for extremely dense or high-power WDM channels, it is accurate for typical wavelength spacings of $0.4 \mathrm{~nm}$ or more.

The material gain $g_{k}(N) \equiv g\left(\nu_{k}, N\right)$ is calculated as in [12, eq. (14)]. Fig. 2 plots the material gain $N$ versus wavelength $\lambda_{k}=c / \nu_{k}$ (with $c$ being the speed of light) using the SOA parameters discussed in the next section.

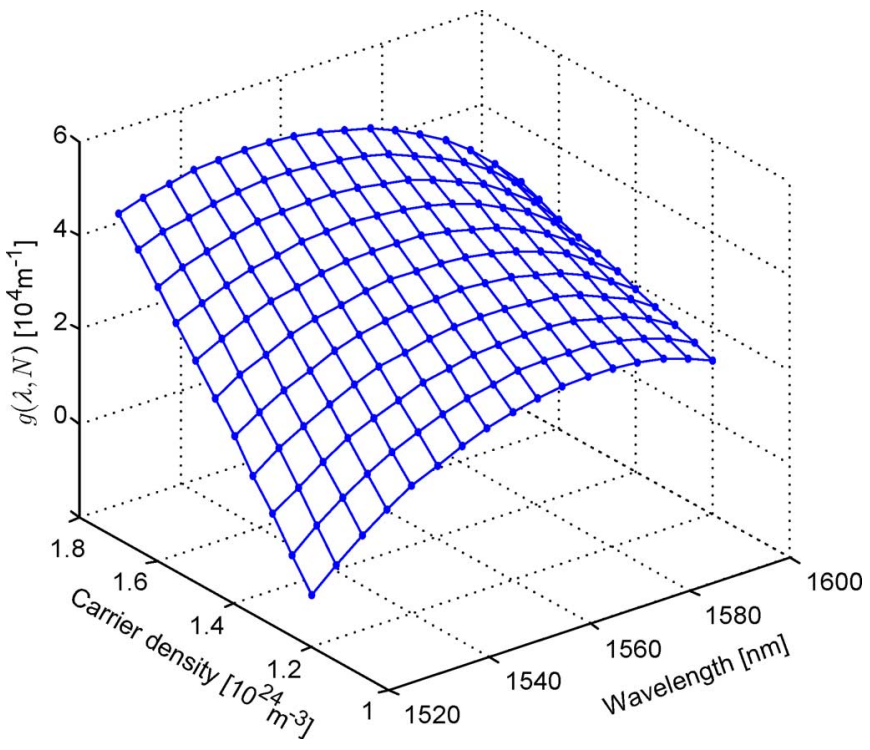

Fig. 2. Gain coefficient $g(\lambda, N)$ versus wavelength and carrier density calculated from ([12, eq. (14)]) with the parameters in Table I.

The time-varying solution of the coupled differential equations (1)-(3) is based on the assumption that the carrier density remains constant during a time step and is achieved by first performing a spatial integration with the carrier density fixed during each time step, followed by a time integration. Steadystate solutions are used as an initial condition for the subsequent time evolution.

We note in closing that the Connelly model also neglects ultrafast phenomena such as $\mathrm{CH}$ and SHB.

\section{B. Parametrization}

In order to fit the experimental results that we obtained with a commercial Optospeed SOA model 1550MRI X1500, we used the SOA parameters provided in [12, Table I], except for a subset of different values reported in Table I in this paper; the most critical of such parameters were determined as follows.

1) The active-region length $L$ was determined by measuring the frequency spacing between two maxima of the gain spectrum ripples: $L=\lambda_{0}^{2} / 2 n_{r} \Delta \lambda$, where $\lambda_{0}$ is the central wavelength $(1550 \mathrm{~nm}), n_{r}$ is the average semiconductor refractive index, and $\Delta \lambda$ is the ripple wavelength spacing.

2) The bandgap energy $E_{g 0}$ was set so that the experimental cutoff wavelength of the gain spectrum (which was about $1605 \mathrm{~nm}$ ) matched the simulated one.

3) The parameters of the carrier-dependent material-loss coefficient [12], i.e.,

$$
\alpha(N(z))=K_{0}+\Gamma K_{1} N
$$

were chosen so that the maximum simulated gain matched the measured one.

4) The active-region thickness and width were set so as to match the experimental and simulated curves of gain as a function of the injection current. 
TABLE I

Values of Parameters USEd in the ConNelly Model, Which Differ From Those in [12]

\begin{tabular}{c||c||c||c}
\hline Symbol & Parameter & Value & Value in [12] \\
\hline \hline $\mathrm{L}$ & Active region length $(\mu \mathrm{m})$ & 1300 & 600 \\
$\mathrm{~d}$ & Active region Thickness $(\mu \mathrm{m})$ & 0.7 & 0.4 \\
$\mathrm{~W}$ & Active region width $(\mu \mathrm{m})$ & 0.7 & 0.4 \\
$K_{g}$ & Bandgap shrinkage coefficient $(\mathrm{eVm})$ & $0.110^{-10}$ & $0.910^{-10}$ \\
$R_{1}$ & Input facet reflectivity & $0.910^{-6}$ & $510^{-5}$ \\
$R_{2}$ & output facet reflectivity & $0.510^{-6}$ & $510^{-5}$ \\
$K_{0}$ & Carrier independent absorption loss coefficient $\left(m^{-1}\right)$ & 6000 & 6200 \\
$K_{1}$ & Carrier dependent absorption loss coefficient $\left(10^{-24} m^{2}\right)$ & 6000 & 7500 \\
$A_{\text {rad }}$ & Linear radiative recombination coefficient $\left(s^{-1}\right)$ & $3.510^{8}$ & $110^{7}$ \\
$B_{\text {rad }}$ & Bimolecular radiative recombination coefficient $\left(m^{3} s^{-1}\right)$ & $410^{-16}$ & $5.610^{-16}$ \\
$A_{\text {nrad }}$ & Linear nonradiative recombination coefficient due to traps $\left(s^{-1}\right)$ & $7.510^{8}$ & $3.510^{8}$ \\
$B_{\text {nrad }}$ & Bimolecular nonradiative recombination coefficient $\left(m^{3} s^{-1}\right)$ & $7.510^{-16}$ & $0.010^{-16}$ \\
$C_{\text {aug }}$ & Auger recombination coefficient $\left(m^{6} s^{-1}\right)$ & $0.210^{-42}$ & $310^{-41}$ \\
$E_{g 0}$ & Bandgap energy $(\mathrm{eV})$ & 0.773 & 0.778 \\
\hline \multicolumn{2}{r}{} & & \\
\hline
\end{tabular}

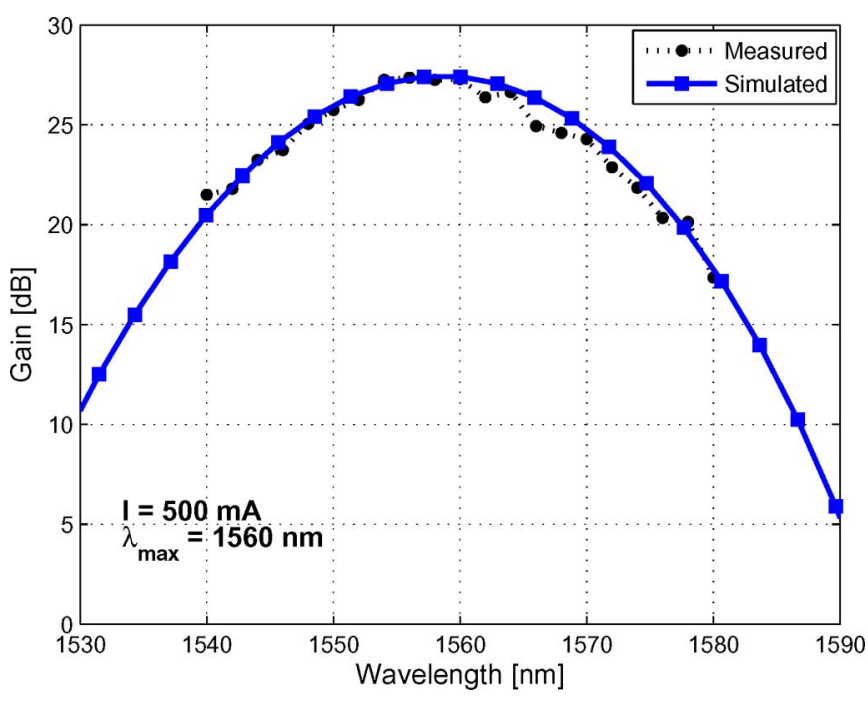

Fig. 3. Fiber to fiber unsaturated gain versus wavelength. Measured (dashed) and simulation (solid) results using Connelly model.

5) The bandgap shrinkage coefficient $K_{g}$ was set so that the peak gain wavelength equals the measured value of $1560 \mathrm{~nm}$ at an injection current of $500 \mathrm{~mA}$.

The aforementioned SOA parameter values were used in all simulations hereafter.

\section{Simulations With Connelly Model}

We present simulation results obtained with the Connelly model and compare them against experimental measurements. The experiment consisted in amplifying a tunable continuous wave $(\mathrm{CW})$ laser whose wavelength was varied around the Optospeed SOA peak gain wavelength. Laser polarization was controlled so as to obtain maximum gain.

1) Unsaturated Gain Spectrum: Fig. 3 shows the simulated and measured unsaturated gain spectra at a signal input power of $-30 \mathrm{dBm}$ and an injection current of $500 \mathrm{~mA}$.

A good match between the simulations and experiments was obtained when using the values of Table I. In the ensuing

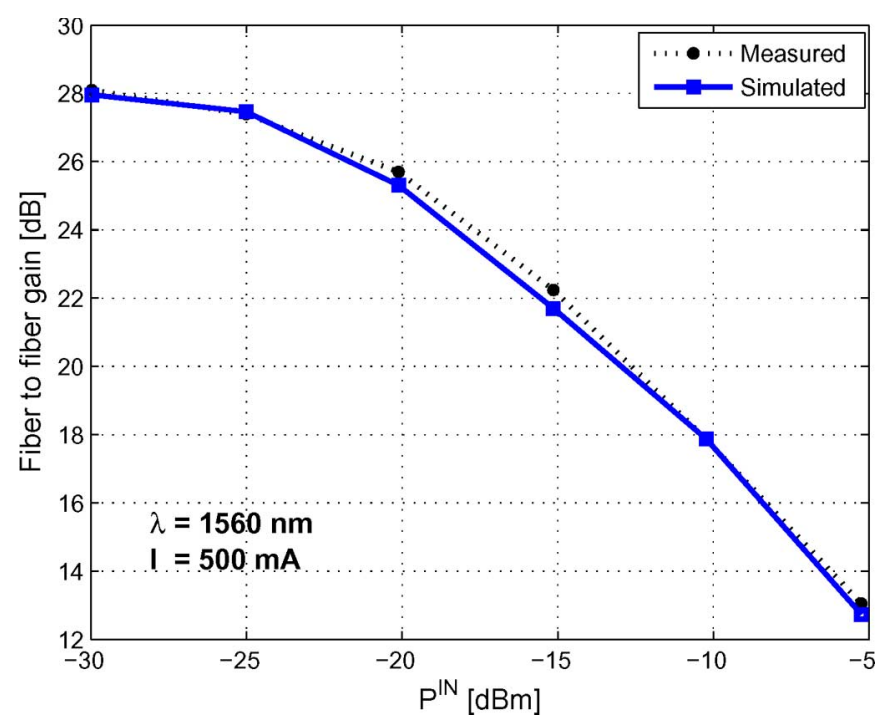

Fig. 4. Fiber to fiber gain versus input optical power. Measured (dashed) and Connelly model (solid).

experiments and simulations, the input signal will be fixed at the gain peak wavelength of $1560 \mathrm{~nm}$.

2) Gain Saturation: Fig. 4 shows the fiber-to-fiber gain as a function of the input power. The wavelength of the input laser was $1560 \mathrm{~nm}$, and the injection current was $500 \mathrm{~mA}$.

3) Dynamic Response: The experimental setup is depicted in Fig. 5. The input laser at $1560 \mathrm{~nm}$ was externally modulated at $1 \mathrm{~Gb} / \mathrm{s}$. The laser power was varied from -25 to $-10 \mathrm{dBm}$ in steps of $5 \mathrm{~dB}$. The measured photoreceiver responsivity was $400 \mathrm{mV} / \mathrm{mW}$. The injection current was $500 \mathrm{~mA}$. Since we are interested in testing the action of the SOA on the propagating signal power in this paper, no optical filter was inserted before detection. $^{1}$

\footnotetext{
${ }^{1}$ The phase response of optical filters can vary nonlinearly with frequency. Signals not precisely centered in the filter passband have the chirp acquired within the SOA transformed into an undesired amplitude modulation, leading to unpredictable pulse shapes that mask the SOA gain dynamics.
} 


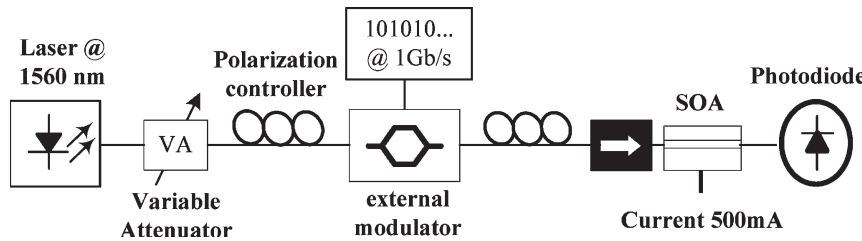

Fig. 5. Experimental setup used to measure the amplified dynamic response.

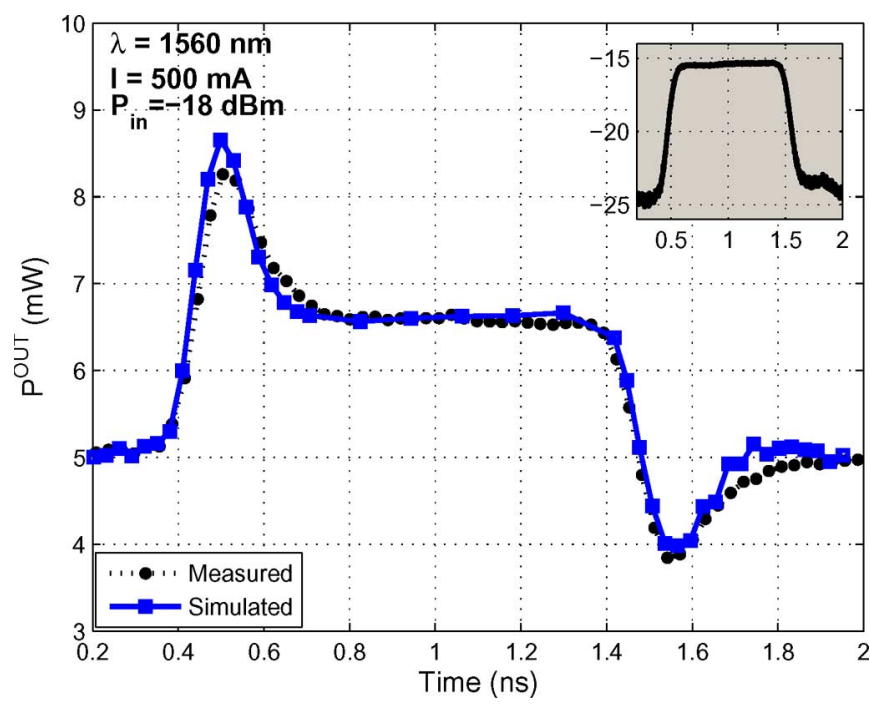

Fig. 6. Response to square wave input (see inset representing optical input power in $\mathrm{dBm}$ ). Measured (dashed) and dynamic Connelly model (solid).

The measured experimental input pulses to the SOA were replicated in the simulator. The length of the input-signal time series was 1350 points over a 2-ns time window. In Fig. 6, we plot the experimental and the simulated output pulses at an input power of $-18 \mathrm{dBm}$. At this power level, the SOA is not heavily saturated by the signal; thus, the ASE-induced saturation significantly contributes to the dynamic response. Fig. 6 demonstrates that the dynamic Connelly model is also able to accurately predict the amplified output pulse shape. Similar results were also obtained for many different input powers and signal wavelengths.

4) Computation Time: The major drawback of the Connelly model is its long execution time. Our Matlab code, which was run on a $3-\mathrm{GHz}$ Intel processor, took about $12 \mathrm{~s}$ to calculate an output bit resolved over 1350 points. Similar calculations for a time series of 50000 points ( 37 bits) took about $432 \mathrm{~s}$. This presents a major limitation when typical Monte Carlo BER estimations are sought, which require transmission of millions of bits. A drastic simplification of the gain dynamics calculation is required in order to significantly decrease execution time. Reduced computation time and the facility of analysis motivate our introduction of the reservoir model.

\section{RESERVOIR MOdel}

We now derive the reservoir model for a traveling-wave SOA (zero facet reflectivity) fed by WDM signals. For $k=$
$1, \ldots, n_{\mathrm{sig}}$, the propagation and carrier density update (1) and (3) become

$$
\begin{aligned}
\frac{d Q_{k}(z, t)}{d z}= & \left\{\left[\Gamma g_{k}(N)-\alpha(N)\right]\right\} Q_{k}(z, t) u_{k} \\
\frac{d N(z, t)}{d t}= & \frac{I}{q V}-R(N)-\frac{\Gamma}{A} \sum_{k=1}^{n_{\mathrm{sig}}} g_{k}(N) Q_{k}(z, t) \\
& -4 \frac{\Gamma}{A} \sum_{j=1}^{n_{\mathrm{ASE}}} g_{j}(N) Q_{j}^{\mathrm{ASE}}(z, t)
\end{aligned}
$$

where $A$ and $V=A L$ are the active waveguide area and volume, respectively, and we introduced the propagation direction variable $u_{k}$, which equals +1 for forward signals and -1 for backward signals. In (5), $2 \cdot Q_{j}^{\mathrm{ASE}}$ stands for an equivalent ASE flux that accounts for the impact of both forward and backward ASE on the carrier-density update equation.

The formal solution of the propagation equation is obtained by multiplying both sides by $u_{k}$, dividing them by $Q_{k}$, integrating both sides in $d z$ from $z=0$ to $z=L$ for each $k$, and obtain an equivalent equation of the form $Q_{k}^{\text {out }}=Q_{k}^{\text {in }} G_{k}$, where the gain

$$
G_{k}=e^{\int_{0}^{L}\left(\Gamma g_{k}(N)-\alpha(N)\right) d z}
$$

is independent of the signal propagation direction. For convenience, we will let

$$
\Gamma g_{k}^{\text {net }}(N) \triangleq \Gamma g_{k}(N)-\alpha(N)
$$

denote the net gain coefficient per unit length in the SOA. Now, define the SOA reservoir as

$$
r(t)=A \int_{0}^{L} N(z, t) d z
$$

which physically represents the total number of carriers in the SOA that are available for conversion into signal photons by the stimulated emission process. If one approximates both the recombination rate and the material gain as linear functions of $N$ [13], i.e.,

$$
\begin{aligned}
R(N) & \cong N / \tau \\
g_{k}(N) & \cong \sigma_{k}\left(N-N_{0 k}\right)
\end{aligned}
$$

where $\tau$ is the fluorescence time and $\sigma_{k}\left[\mathrm{~m}^{2}\right]$ and $N_{0 k}\left[\mathrm{~m}^{-3}\right]$ are wavelength-dependent fitting coefficients, then one obtains from (4) and (6)

$$
G_{k}=\exp \left[\int_{0}^{L} \Gamma g_{k}^{\text {net }}(N) d z\right]=\exp \left[a_{k}\left(r(t)-r_{0 k}\right)\right]
$$

where

$$
\begin{aligned}
a_{k} & =\Gamma\left(\sigma_{k}-K_{1}\right) / A \\
r_{0 k} & =\left(\Gamma \sigma_{k} N_{0 k}+K_{0}\right) L / a_{k}
\end{aligned}
$$


are two dimensionless parameters. In addition, one can multiply both sides of the second equation in (5) by $A$ and integrate in $d z$ to obtain

$$
\begin{aligned}
\frac{d r(t)}{d t}=\frac{I}{q}-\frac{r(t)}{\tau} & -\sum_{k=1}^{n_{\text {sig }}} \int_{0}^{L} \Gamma g_{k}(N(z)) Q_{k}(z) d z \\
& -4 \sum_{j=1}^{n_{\mathrm{ASE}}} \int_{0}^{L} \Gamma g_{j}(N(z)) Q_{j}^{\mathrm{ASE}}(z) d z .
\end{aligned}
$$

For the time being, the contribution of ASE will be neglected. It will be tackled in Section III-B. Now, integrating in $d z$ both sides of the first equation in (5) gives

$$
\int_{0}^{L} \Gamma g_{k}(N) Q_{k} d z=\left(Q_{k}^{\text {out }}-Q_{k}^{\text {in }}\right)+\int_{0}^{L} \alpha(N) Q_{k} d z .
$$

The Appendix discusses the approximation involved in dropping the second term on the right-hand side of (13), which physically represents the signal photons lost through scattering within the SOA. If such a scattering loss term can be dropped, then substituting in (12), one gets the "reservoir dynamic equation" given by

$$
\frac{d r(t)}{d t}=\frac{I}{q}-\frac{r(t)}{\tau}-\sum_{k=1}^{n_{\mathrm{sig}}} Q_{k}^{\text {in }}\left(e^{a_{k}\left(r(t)-r_{0 k}\right)}-1\right)
$$

where gain $G_{k}$ (10) takes the scattering losses into account. Since the scattering loss term is positive, the reservoir resulting from (14) is an overestimation of the actual reservoir, as quantified in the Appendix.

Note that the reservoir dynamic equation is quite similar to the EDFA reservoir equation [21] and, together with gain (10), builds the block diagram shown in Fig. 1.

As discussed in [13], one can easily show that once reservoir $r(t)$ is known, the cumulated phase of the $k$ th propagating signal at the output of the SOA can be obtained as

$$
\phi_{k}^{\text {out }}(t)=-\frac{1}{2} \alpha_{H} \frac{\Gamma \sigma_{k}}{A}\left(r(t)-N_{0 k} V\right)
$$

where $\alpha_{H}$ is the "linewidth enhancement factor." Therefore, it is possible to correctly take into account chirp-related distortions that are induced by dispersive optical components that follow the SOA.

\section{A. Extraction of Reservoir Parameters From Connelly Model}

We next explain how to extract the fitting parameters of the gain linearization in (9) from the Connelly gain $g(\lambda, N)$, whose plot versus wavelength and carrier density was already given in Fig. 2 for our Optospeed SOA. A plot of $g_{k}^{\text {net }}(\lambda, N)$ would have a similar form; in particular, a rigid shift downward would result if $K_{1}=0$, i.e., if $\alpha$ did not depend on $N$.

Fig. 7 gives a slice of the surface in Fig. 2 at a wavelength of $1560 \mathrm{~nm}$, which was plotted over a wide range of carrier density $N$. As shown, a linear approximation of the gain coefficient is well justified especially as the physically achievable range of

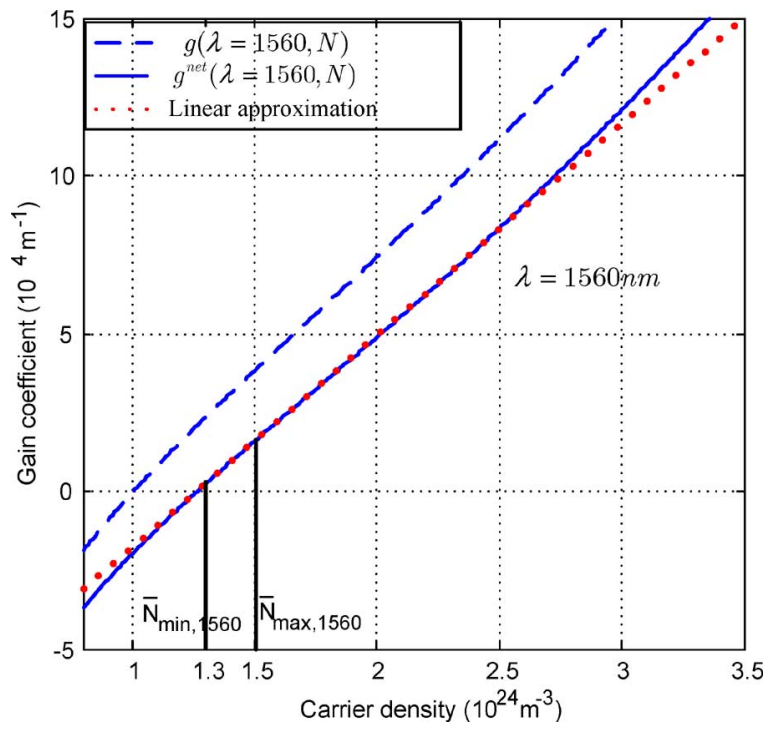

Fig. 7. Connelly gain coefficient $g$ (dashed) and net gain coefficient $g^{\text {net }}$ in (7) (solid) versus carrier density $N$ for $\lambda=1560 \mathrm{~nm}$. SOA parameters as in Table I. Dotted is the linear approximation used in the reservoir model.

carrier densities is much smaller than the range shown. Our task is now to provide good estimates of the wavelength-dependent coefficients $\sigma_{k}$ and $N_{0 k}$ in (9).

First, we identify the achievable range of $N$ over which we will restrict our linear fit. To this aim, using the steady-state Connelly model, we calculated the maximum and minimum values of the "average carrier density," i.e.,

$$
\bar{N} \triangleq \frac{1}{L} \int_{0}^{L} N(z) d z=\frac{r}{V}
$$

which were obtained for the extreme cases of a single input signal at very low $(-40 \mathrm{dBm})$ and very high $(0 \mathrm{dBm})$ input power at $1560 \mathrm{~nm}$. These extremes cover the small-signal regime and saturation at an injection current of $500 \mathrm{~mA}$. Equation (5) without ASE was used to find $N(z)$ at steady state $(d N / d t=$ 0 ) for a small signal and saturation at $\lambda_{k}$. The carrier density was integrated across $z$ to give the extreme values $\bar{N}_{\max , k}$ and $\bar{N}_{\text {min, },}$, which are depicted in Fig. 7. The process was repeated at each wavelength from 1450 to $1600 \mathrm{~nm}$ in intervals of $5 \mathrm{~nm}$. The parameters of the gain coefficient linear fit were then extracted from the extreme values as follows:

$$
\sigma_{k}=\frac{g_{\max , k}-g_{\min , k}}{N_{\max , k}-N_{\min , k}}, \quad N_{0, k}=N_{\max , k}-\frac{g_{\max , k}}{\sigma_{k}}
$$

where $g_{\max , k} \triangleq g\left(\lambda_{k}, \bar{N}_{\max , k}\right)$ and $g_{\min , k}$ is similarly defined. In Fig. 8, we provide the wavelength dependence of the extracted fitting parameters $\sigma_{k}$ and $N_{0, k}$ for our Optospeed SOA.

Once the linearized gain parameters are calculated, we can investigate the steady state and the dynamic behavior predicted by the reservoir model and, as explained in the Appendix, look for the value of $\tau$ that best fits the steady-state and dynamic experimental curves. However, before doing so, the fundamental role of spontaneous emission in the rate equation must be properly accounted for. 


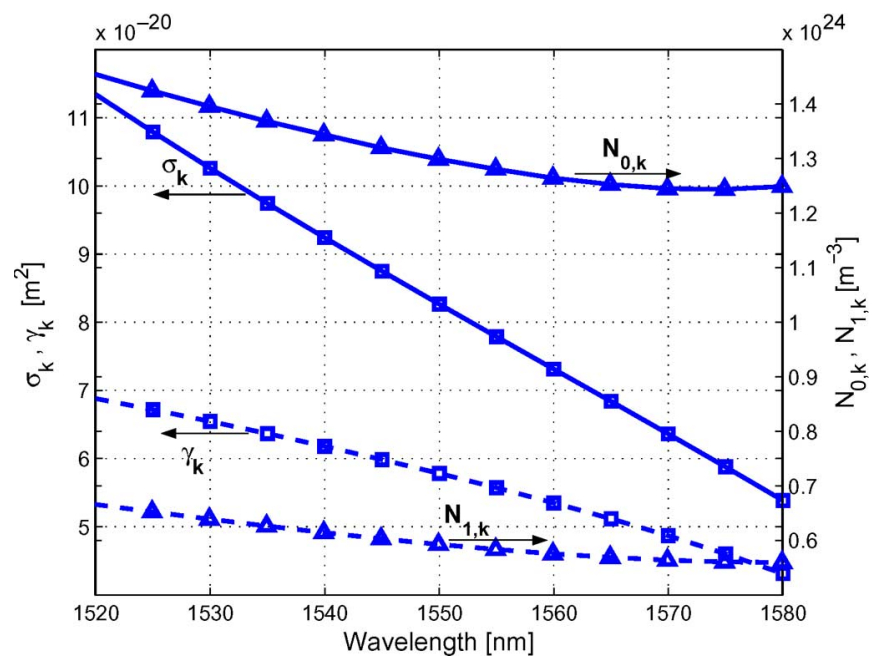

Fig. 8. Coefficients $\sigma_{k}$ (squares solid) and $N_{0, k}$ (triangle solid) of the linearization of the gain coefficient $g$ versus wavelength for our Optospeed SOA. Also shown are the coefficients $\gamma_{k}$ and $N_{1, k}$ of the linearization of the emission gain coefficient $g^{\prime}$ (see Section III-B).

\section{B. Including ASE}

We now take into account the ASE-induced saturation term in (5) that was neglected in the previous section. The ASE flux at $z$ is obtained by solving the propagation (2) with zero initial condition [27]:

$$
Q_{j}^{\mathrm{ASE}}(z)=G_{j}(z) \int_{0}^{z} \frac{R_{\mathrm{sp}, j}(N(s))}{G_{j}(s)} d s
$$

where $G_{j}(z)=\exp \left[\int_{0}^{z} \Gamma g_{j}^{\text {net }}\left(N\left(z^{\prime}\right)\right) d z^{\prime}\right]$ is the gain from 0 to $z$. If, for this calculation, we assume that the carrier density is constant along $z$ at the average carrier density $\bar{N}=r / V$, then the preceding equation simplifies to

$$
Q_{j}^{\mathrm{ASE}}(z) \cong \frac{R_{\mathrm{Sp}, j}(\bar{N})}{\Gamma g_{j}^{\mathrm{net}}(\bar{N})}\left(e^{\Gamma g_{j}^{\mathrm{net}}(\bar{N}) z}-1\right)
$$

Such an expression can now be used to evaluate the ASE integrals neglected in (12) in the previous section, i.e.,

$$
\begin{aligned}
\int_{0}^{L} \Gamma g_{j}(N) Q_{j}^{\mathrm{ASE}}(z) d z \cong \frac{g_{j}(\bar{N})}{g_{j}^{\text {net }}(\bar{N})} \cdot \frac{R_{\mathrm{sp}, j}(\bar{N})}{\Gamma g_{j}^{\text {net }}(\bar{N})} \\
\cdot(G(r)-1-\ln G(r))
\end{aligned}
$$

where $G(r)=\exp \left\{\Gamma g_{j}^{\text {net }}(\bar{N}) L\right\}$ is the gain and is a function of the reservoir only. Note that the fraction $g_{j} / g_{j}^{\text {net }}$ on the righthand side of (20) also appeared in the treatment of the signals in the Appendix. It approaches one when the scattering-loss coefficient is small compared with the gain coefficient, and in the following, it will be considered equal to one in the same way we dealt with the signals in the previous section.

The fraction in (19) represents the spontaneous emission factor $n_{\mathrm{sp}, j}$ at the $j$ th wavelength of the SOA multiplied by the resolution bandwidth of the ASE channels $\Delta \nu_{\mathrm{ASE}}$. Connelly [12, eq. (40)] points out that $R_{\mathrm{sp}, j}(N)=\Gamma g_{j}^{\prime}(N) \Delta \nu_{\mathrm{ASE}}$, where $g_{j}^{\prime}>g_{j}$ is the emission gain coefficient [12, eq. (16)], which may significantly differ from the gain coefficient $g_{j}$ at shorter wavelengths. If we linearize $g_{j}^{\prime}(N) \cong \gamma_{j}\left(N-N_{1 j}\right)$ and use the linearization (9) of $g_{j}$, then we get

$$
n_{\mathrm{sp}, j}=\frac{R_{\mathrm{sp}, j}(\bar{N}) / \Delta \nu_{\mathrm{ASE}}}{\Gamma g_{j}^{\text {net }}(\bar{N})} \cong \frac{\Gamma \gamma_{j}\left(r-r_{1 j}\right)}{A a_{j}\left(r-r_{0 j}\right)}
$$

where $r_{1 j} \triangleq N_{1 j} V$. As a dimensional check, $\gamma_{j}$ and $A$ are measured in $\left[\mathrm{m}^{2}\right]$, while $a_{j}$ is dimensionless so as to correctly obtain a dimensionless $n_{\mathrm{sp}, j}$.

Fig. 8 also shows the values of the wavelength-dependent coefficients $\gamma_{j}$ and $N_{1 j}$ in the linearization of $g^{\prime}$, which were obtained using exactly the same procedure that yields the linearization coefficients of $g$ detailed in Section III-A.

Finally, using (12), (20), and (21), the reservoir dynamic equation including ASE becomes

$$
\begin{aligned}
\frac{d r(t)}{d t} & =\frac{I}{q}-\frac{r(t)}{\tau}-\sum_{k=1}^{n_{\mathrm{sig}}} Q_{k}^{\mathrm{in}}\left(e^{a_{k}\left(r(t)-r_{0 k}\right)}-1\right) \\
& -4 \Delta \nu_{\mathrm{ASE}} \sum_{j=1}^{n_{\mathrm{ASE}}} n_{\mathrm{sp}, j}(r)\left(G_{j}(r)-1-\ln G_{j}(r)\right) .
\end{aligned}
$$

It is worth noting that a similar equation is found for the case of EDFAs in [29, eq. (13)]. The received ASE flux $Q_{j}^{\mathrm{ASE}}(L)$, finally, is calculated from (19) and (21).

Since this equation still partly neglects some contributions of the effect of scattering loss, as discussed in the Appendix, we found it convenient to tune the value of the fluorescence time $\tau$ in the reservoir model so as to best match the dynamic and static behaviors of the Optospeed SOA used in the experiments. A value close to $\tau=310 \mathrm{ps}$ was found to give an excellent fit in the cases that we analyzed.

As a first example of the validity of (22), we calculated both with the Connelly model and with the reservoir model the total received ASE power when a single nonreturn-tozero modulated channel at $1560 \mathrm{~nm}$, with a 0101 modulating sequence at $1 \mathrm{~Gb} / \mathrm{s}$, was fed to the $\mathrm{SOA}$ with an input power of $-18 \mathrm{dBm}$. Fig. 9 shows the total received ASE power as a function of time for both models, with a satisfactory agreement. Extensive comparisons will be presented in Section IV.

\section{Multistage Reservoir Model}

The multistage reservoir model consists of subdividing the SOA into several cascaded sections or "stages," each characterized by its own reservoir (Fig. 10). Let $n_{s}$ be the number of stages. Then, the reservoir equation for each stage $i$ is

$$
\begin{gathered}
\frac{d r_{i}}{d t}=\frac{I}{q}-\frac{r_{i}}{\tau}-\sum_{k=1}^{n_{\mathrm{sig}}} Q_{k, i}^{\mathrm{in}}\left(G_{k}\left(r_{i}\right)-1\right)-\sum_{j=1}^{n_{\mathrm{ASE}}} Q_{j, i}^{\mathrm{ASE}, \mathrm{in}}\left(G_{j}\left(r_{i}\right)-1\right) \\
-4 \Delta \nu_{\mathrm{ASE}} \sum_{j=1}^{n_{\mathrm{ASE}}} n_{\mathrm{sp}, j}\left(r_{i}\right)\left(G_{j}\left(r_{i}\right)-1-\ln \left(G_{j}\left(r_{i}\right)\right)\right)
\end{gathered}
$$

where $r_{i}$ is the reservoir of the $i$ th stage with length $L_{i}=L / n_{s}$ and $G_{k}\left(r_{i}\right)$ is its gain given in (10) and (11) (where $L_{i}$ is 


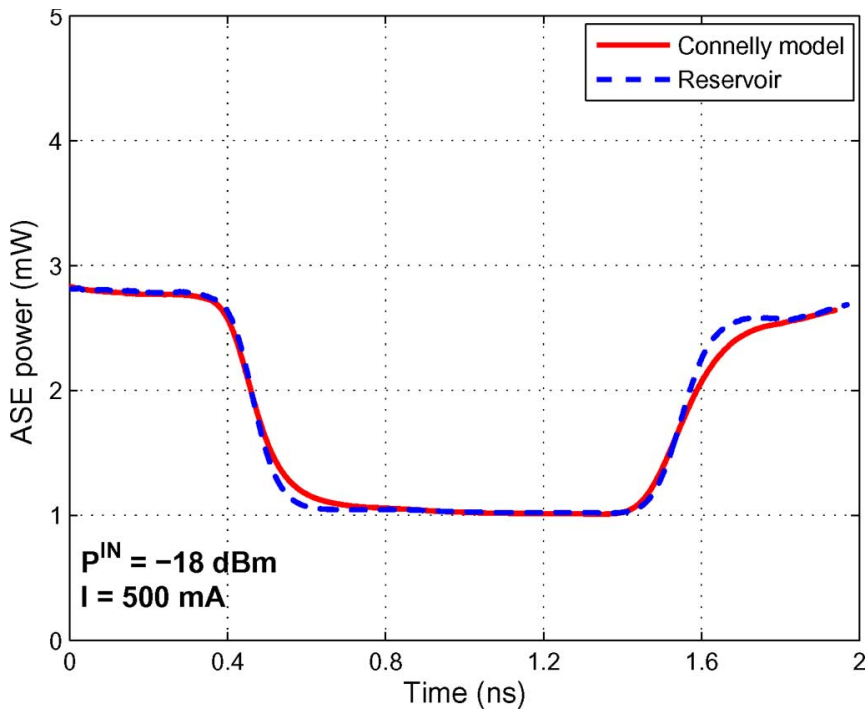

Fig. 9. Variation of the total output ASE power for a square input pulse train (inset of Fig. 6); simulation results with dynamic Connelly model (solid) and with reservoir model including ASE (dashed).

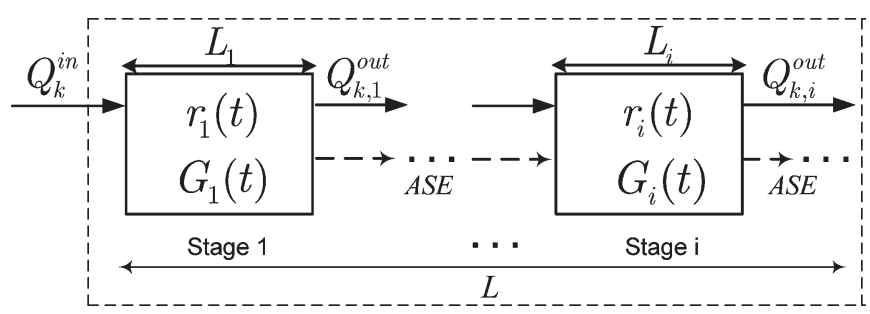

Fig. 10. Multistage reservoir model.

used instead of $L$ ), and $n_{\mathrm{sp}, j}$ is the spontaneous emission factor in (21). For the signal channels, the flux $Q_{k, i+1}^{\text {in }}$ input to the $(i+1)$ th stage is the output flux of the $i$ th stage, which is in turn equal to the $i$ th reservoir gain $G_{k}\left(r_{i}\right)$ multiplied by its input flux $Q_{k, i}^{\mathrm{in}}$. For the ASE channels, the first-stage input flux is zero. The output ASE of one stage becomes an input ASE signal to the next stage, which is accounted for in (23) by the second summation term. The third summation term is, as usual, the ASE generated inside stage $i$. Considering forward ASE only has the advantage of simplicity, but the approximation brought into a multistage scenario is evident: Each stage is saturated by forward ASE from the upstream stages. Modeling the SOA with multiple stages is similar to the algorithm used in the spaceresolved models, which provide the carrier-density evolution $N\left(t, z_{i}\right)$ at discrete positions $z_{i}$ along the SOA. Hence, the multistage reservoir model is expected to give similar results to the Connelly model.

\section{Reservoir Model With Single-Channel ASE}

Consider the single-stage reservoir model. In order to further speed up calculations, we now introduce a single fictitious CWinput ASE channel. Once its wavelength is fixed, the power of such a CW channel should be chosen so that the time behavior of reservoir $r(t)$ in a noiseless SOA is as close as possible to $r(t)$ in the actual SOA that is saturated by signals and ASE. We call such an input channel the "ASE depleting channel"
TABLE II

Simulation Times for 1350 Time Points for A Multistage Reservoir With Multiple ASE or Single ASE Channels

\begin{tabular}{c||c||c}
\hline Stages & 20 ASE channels & 1 ASE channel \\
\hline \hline 1 & $1.32 s$ & $0.58 \mathrm{~s}$ \\
2 & $1.54 \mathrm{~s}$ & $1.11 \mathrm{~s}$ \\
5 & $4.26 \mathrm{~s}$ & $2.75 \mathrm{~s}$ \\
10 & $7.74 \mathrm{~s}$ & $5.30 \mathrm{~s}$ \\
\hline
\end{tabular}

since the photons used to amplify this channel are lost and not available to amplify the signal channels.

In this case, the reservoir (22) simplifies as

$$
\frac{d r}{d t}=\frac{I}{q}-\frac{r}{\tau}-\sum_{k=1}^{n_{\mathrm{sig}}} Q_{k}^{\mathrm{in}}\left(G_{k}(r)-1\right)-Q_{\mathrm{ASE}}^{\mathrm{in}}\left[G_{\mathrm{ASE}}(r)-1\right]
$$

where $G_{\mathrm{ASE}}$ is the gain at the wavelength of the ASE depleting channel. After arbitrarily fixing the ASE wavelength, we determined the most appropriate value of $Q_{\mathrm{ASE}}^{\mathrm{in}}$ by minimizing the mean square error in the dynamic step response of the SOA with respect to the predictions of the Connelly model. The trick of the ASE depleting channel can also be extended to the multistage case.

\section{RESUlTS}

The purpose of this paper is to demonstrate that calculations using the SOA reservoir model are much faster than the spaceresolved Connelly model and hence, are suitable for Monte Carlo simulations. We also demonstrate that using the correct wavelength-dependent parameters, the reservoir model is sufficiently accurate. In this section, we first compare the computation speed of both models. Then, we assess the accuracy of the reservoir models that were developed in the previous sections by comparing gain spectrum, gain saturation, and dynamic response with the predictions of our experiments.

\section{A. Calculation Speed}

We present the calculation times required for different models, namely, the dynamic Connelly model presented in Section II, the reservoir model with multiple ASE channels in Section III-B, and the reservoir model with a single ASE channel in Section III-D. For the reservoir model, we determined the computation time for a single-stage SOA, as well as three multistage SOAs (two, five, and ten stages). The calculation times in Table II refer to the response to a single input pulse with a duration of 2 ns that was resolved over 1350 temporal points. As a reference, the execution time for the Connelly model was $11.95 \mathrm{~s}$. The calculation times in Table III refer to the response to a string of multiple pulses with the same time step as before, for a total of 50000 temporal points. As a reference, the execution time for the Connelly model was 432.54 s. In the Connelly model, we always used a space resolution of $43.33 \mu \mathrm{m}$, with the ASE resolved over 30 channels in bins of $2.5 \mathrm{~nm}$ each, which were symmetrically arranged around the gain peak.

As shown, the reservoir model with single-stage ASE is always the fastest model. The simulation is 20 times faster 
TABLE III

Simulation Times for 50000 Time Points for a Multistage Reservoir With Multiple ASE or Single ASE ChanNels

\begin{tabular}{c||c||c}
\hline Stages & 20 ASE channels & 1 ASE channel \\
\hline \hline 1 & 40.78 & 19.01 \\
2 & 78.94 & 38.17 \\
5 & 196.51 & 94.89 \\
10 & 389.33 & 189.7
\end{tabular}

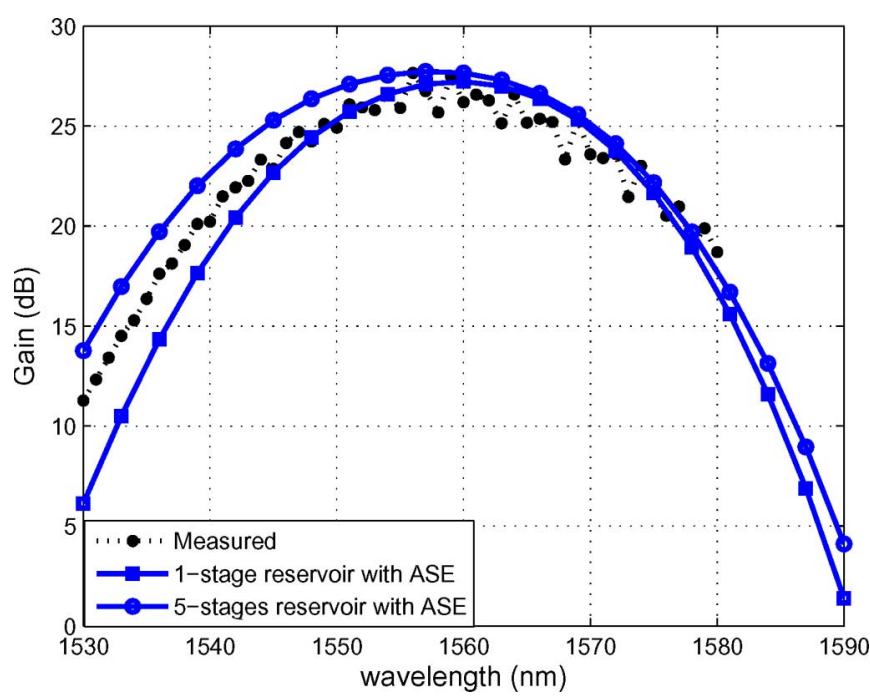

Fig. 11. Fiber to fiber gain spectrum versus wavelength. Measured (dashed) and simulation (solid) results using the single (squares) and five-stage (circles) reservoir with 20 ASE channels.

than the Connelly model when a single ASE channel is used. However, when several reservoir stages are used, the calculation speed of the single-ASE model becomes of the same order as that of the multiple-ASE case. In this case, the use of multiple ASE channels is better for accuracy.

The improvement in computation time in all reservoir models with respect to the Connelly model is predicted to significantly increase when increasing the number of propagated WDM signal channels.

\section{B. Single-Stage Reservoir With ASE}

1) Gain Spectrum: Fig. 11 shows both simulated (solid lines with markers) and experimental fiber-to-fiber (dasheddotted line) gain versus wavelength. The input laser power was $-25 \mathrm{dBm}$. We can see a reasonable match between simulations and experiments. A slight gap between simulation and experiment is observed at shorter wavelengths. The peak gain wavelength was the same in both simulations and experiment. We also see that the five-stage reservoir model is slightly more accurate than the single-stage reservoir one.

2) Gain Saturation: Fig. 12 shows both simulated and experimental fiber-to-fiber gains versus input power at a signal wavelength of $1560 \mathrm{~nm}$. We see that the simulations reasonably predict the small-signal gain. A slight discrepancy is observed when saturation sets in. This is attributed to the fact that the ratio $g_{k} / g_{k}^{\text {net }}$ is larger than one in deep saturation since the denominator tends to zero. In such cases, it is preferable to include the term $g_{k}(r) / g_{k}^{\text {net }}(r)$ in the reservoir equation rather

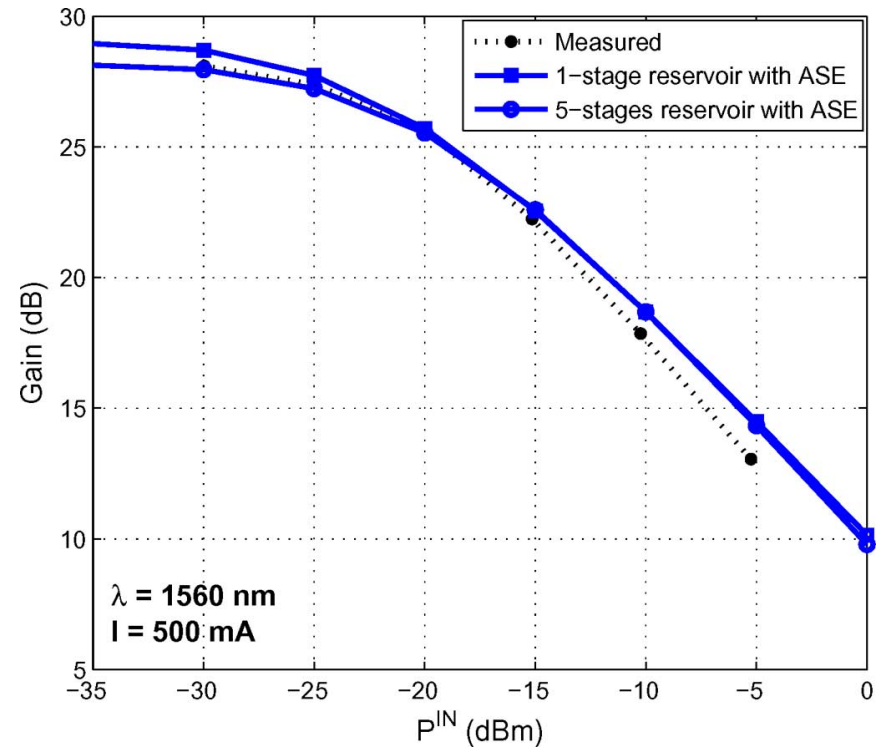

Fig. 12. Fiber to fiber gain versus input optical power. Measured (dashed) and simulation (solid) results using the single (squares) and five-stage (circles) reservoir with 20 ASE channels.

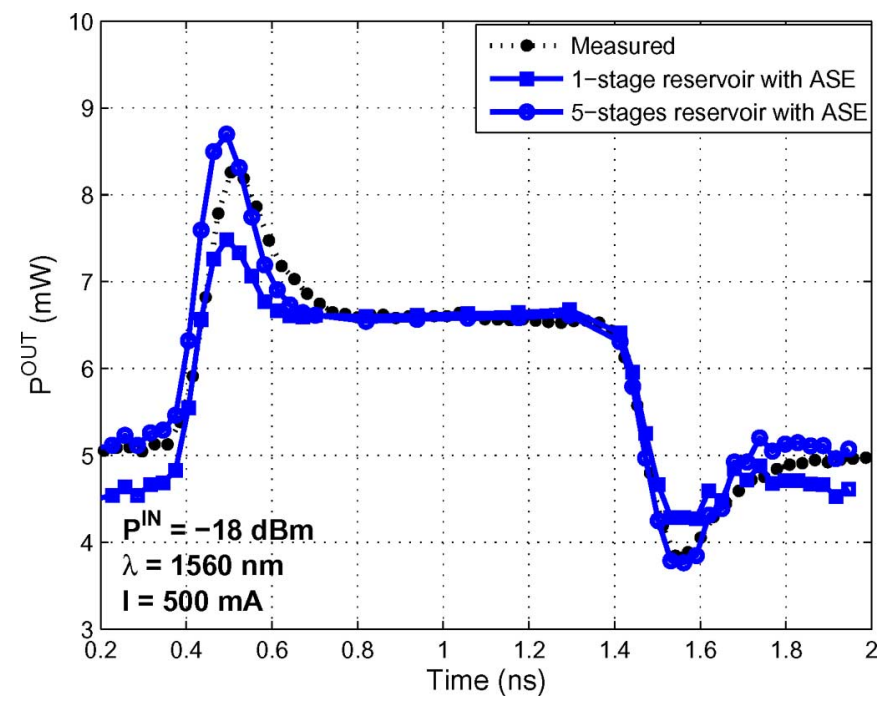

Fig. 13. Response to square wave input. Measured (dashed) and simulation (solid) results using the single (squares) and five-stage (circles) reservoir with 20 ASE channels.

than set it to 1 and play with the fitting parameter $\tau$, as we did in this paper. Here again, we see that the five-stage reservoir is closer to the experimental data.

3) Dynamic Response: Fig. 13 shows the simulated (solid with squared markers) and experimental (dashed) response in milliwatts to a square-wave input (see inset in Fig. 6). Simulations include the ASE total detected power, which plays a fundamental role in the reservoir equation, since it partially saturates the amplifier, hence reducing the amplifier gain. We see that the CW levels (zero and one levels) are well predicted in Fig. 13. This suggests that the approximation that we used to calculate the ASE power is valid, although the simulated and experimental output pulses are slightly different. We see that the pulse's overshoot and undershoot are better predicted by the five-stage reservoir model. 


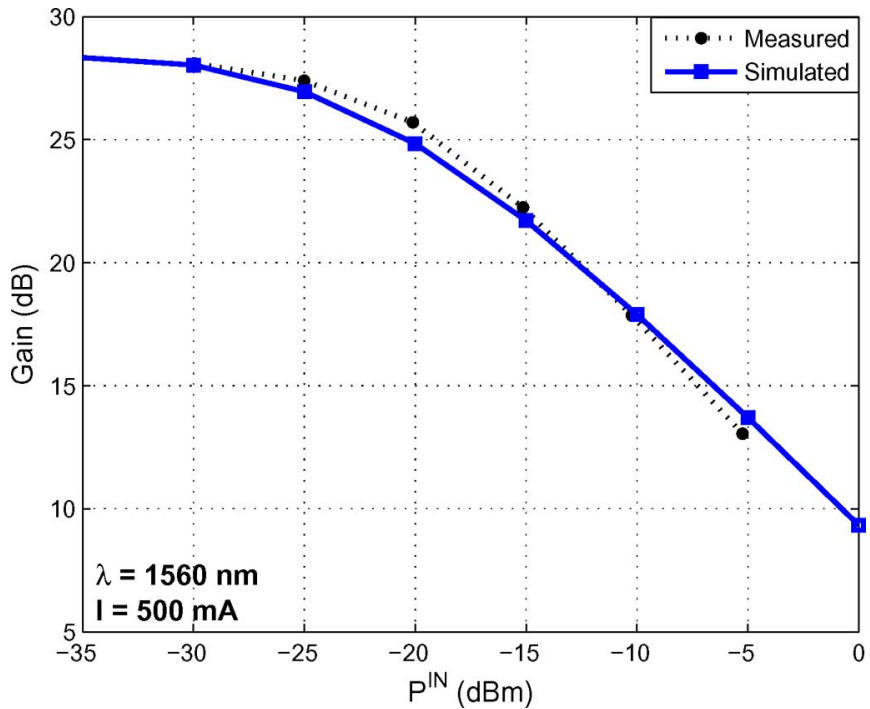

Fig. 14. Fiber to fiber gain versus optical input power. Measured (dashed) and simulated (solid) results using a three-stage reservoir with single channel ASE.

\section{Multistage Reservoir With ASE}

Figs. 11-13 show the gain spectrum, gain saturation at $1560 \mathrm{~nm}$, and the output pulse power for the five-stage (solid with circle markers) reservoir with ASE, respectively. The parameters used in the simulations are the same as those considered in the single-stage reservoir model. Increasing the stage number beyond five does not increase the accuracy, which might be attributed to the neglect of ASE that propagates backward across the stages. In these figures, we see that the dynamic and steady-state fits are more accurate than those in the single stage. Particularly, the simulated gain spectrum shape (Fig. 11) is closer to the experimental one compared with the single-stage reservoir. Moreover, the overshoot and undershoot of the output pulse are much closer to the experimental one. However, this precision comes at the price of simulation speed: The larger the number of stages, the longer the execution time (see Tables II and III). An advantage of the multistage model is that it allows trading execution time for precision, eventually reaching a comparable precision (and a comparable computational burden) as the space-resolved Connelly model.

\section{Multistage Reservoir With ASE Depleting Channel}

In order to fit the experimental results, we arbitrarily fixed the ASE-depleting-channel wavelength at $1520 \mathrm{~nm}$ and then found the value of its input flux, which gave the minimum mean square error fit with the prediction of the Connelly model.

As shown in Figs. 14 and 15, the simulation results are not far from the experimental ones, but they are less accurate than those in the multichannel ASE case. To investigate the dynamic response, we cascaded three reservoir stages and propagated both the signal and the ASE depleting channel. We note from the figures that simulations fit measurements in a way comparable to the multistage reservoir with ASE, which proves the effectiveness of the ASE-depleting-channel approach. Moreover, the advantage of this approach is the computation speed. In fact, ASE-depleting-channel simulations

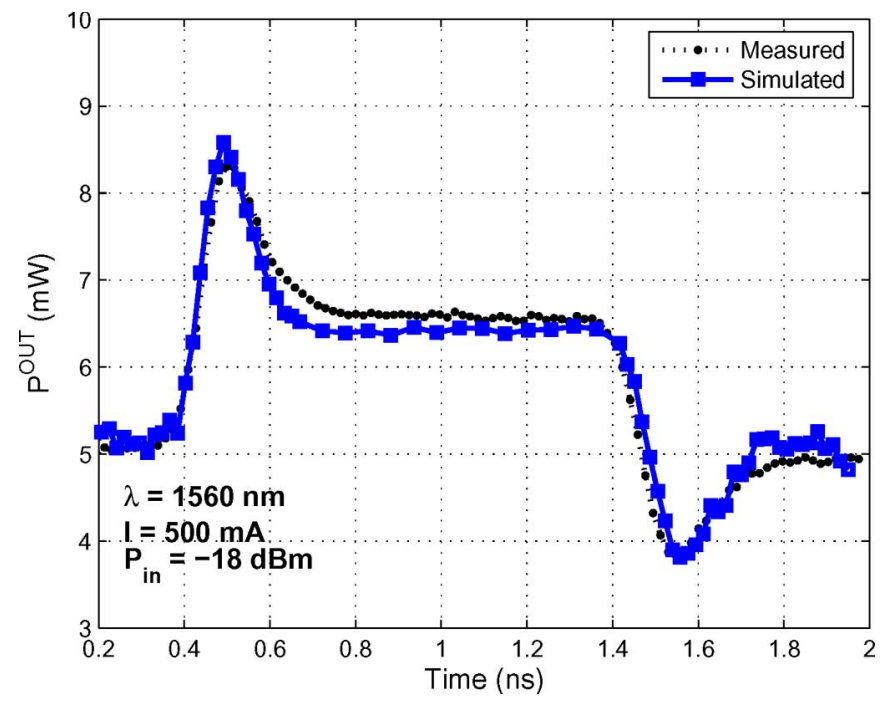

Fig. 15. Response to a square wave input. Measured (dashed) and simulated (solid) results using a three-stage reservoir with ASE depleting channel.

are twice as fast as those for the multichannel ASE case (see Tables II and III). The use of more than three stages does not improve accuracy.

\section{E. WDM Amplification}

In order to verify the efficiency of our model for a wider range of simulation scenarios, we investigated the case of WDM-signal amplification. We recall that both the Connelly and the reservoir models are not able to reproduce carrierinduced nonlinear effects such as FWM and XPM and can only model the effects of carrier-induced self-gain modulation and XGM.

Fig. 16 shows the measured power at the output of our Optospeed SOA as well as simulation results using the Connelly model and (a) a one-stage reservoir model and (b) a three-stage model, in which the fluorescence $\tau$ was set at the value of 360 ps to best match the measurements. The SOA was fed with four synchronously OOK-modulated WDM signals with a wavelength spacing of $3 \mathrm{~nm}\left(\lambda_{1}=1550 \mathrm{~nm}, \lambda_{2}=1553 \mathrm{~nm}\right.$, $\lambda_{3}=1556 \mathrm{~nm}$, and $\left.\lambda_{4}=1559 \mathrm{~nm}\right)$. The SOA output is optically filtered so that the ASE is eliminated, and the desired channel is selected. The optical filter is $1.2 \mathrm{~nm}$ wide, so its effect on the pulse shape is negligible at an experimental bit rate of $1 \mathrm{~Gb} / \mathrm{s}$. The average input power of each channel is $-20 \mathrm{dBm}$ (experimentally, lower input power showed noisy pulses). Under such conditions, we observe a good match between the measurements and the Connelly model predictions. A reasonable match is also obtained between the experiment and the reservoir models. However, we verified that at lower input powers, the simulations give a less exact fit. The lack of accuracy during the transients is due to the linear approximations of the gain and recombination rate. Note the different slopes of measured and simulated pulses after the overshoot in Fig. 15. We believe the reason for this to lie in the linear approximation of $R(N)$ is when the signal reaches a maximum (and the carrier density reaches 


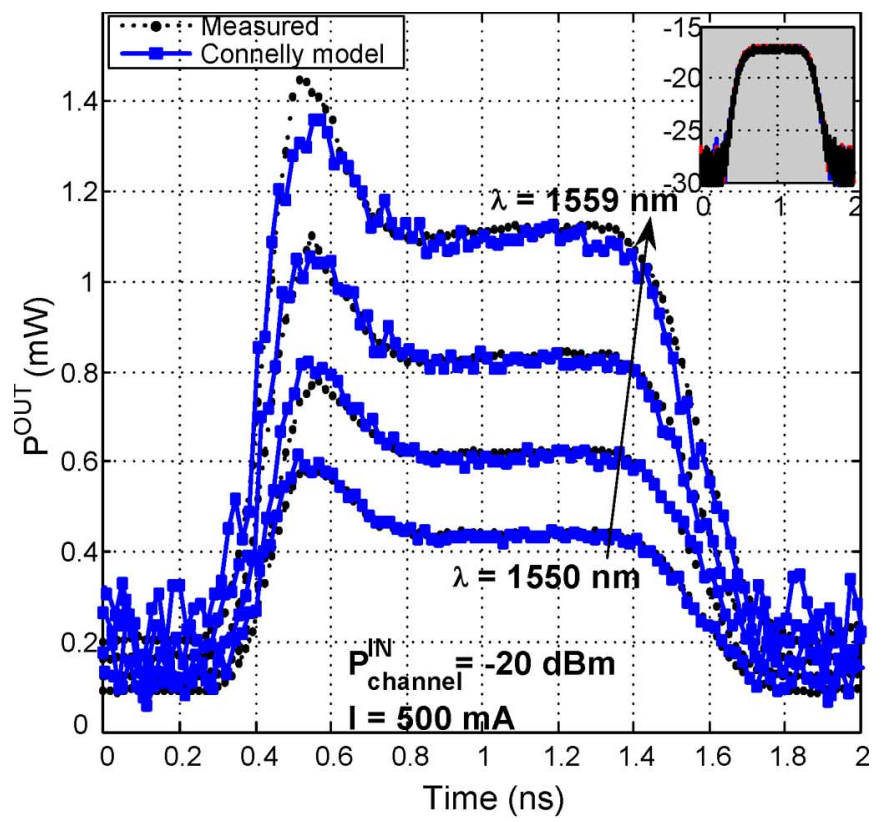

(a)

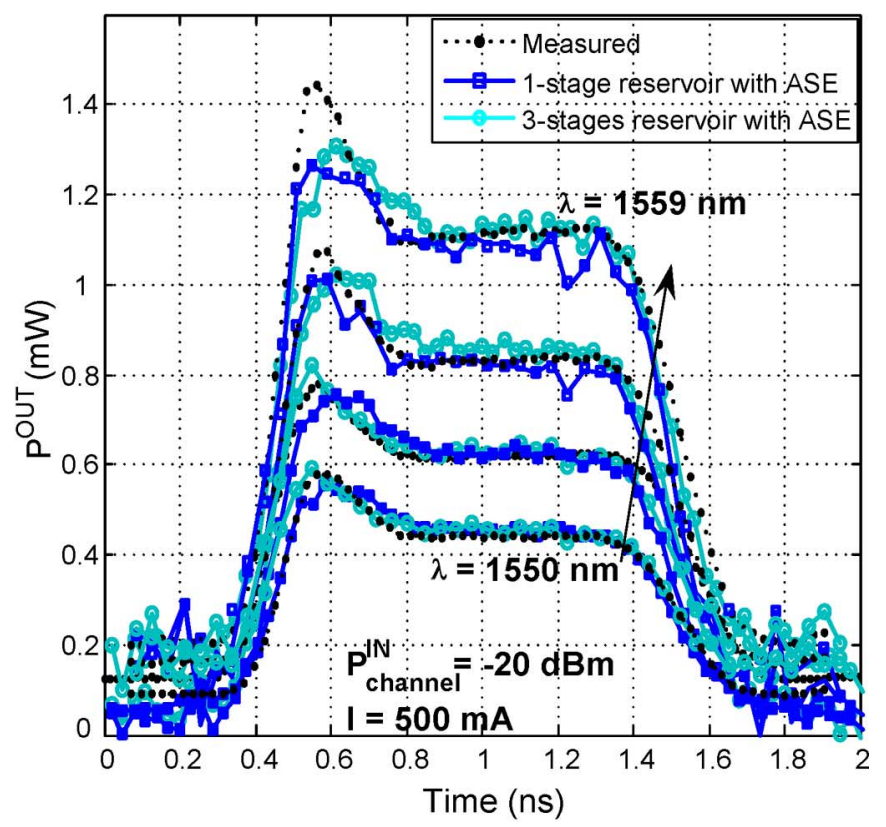

(b)

Fig. 16. Response of four WDM channels (with a spacing of $3 \mathrm{~nm}$ ) to a square wave input (see inset showing input optical powers in $\mathrm{dBm}$ ). (a) Measured (dashed) and dynamic Connelly model (solid). (b) Measured (dashed), onestage reservoir with single channel ASE (solid with squares) and three-stage reservoir with single channel ASE(solid with circles).

a minimum), the actual time constant of the SOA is larger than that employed in (9). Moreover, ultrafast phenomena (neglected in this paper) will have an increasing impact for overshoots and undershoots on the order of a few picoseconds.

\section{CONCLUSION}

A novel state-variable SOA model that is amenable to block diagram implementation for WDM applications and with fast execution times was presented and discussed. We called the novel model the reservoir model, in analogy with similar blockoriented models for EDFAs and Raman amplifiers. While ASE self-saturation can be simply included in the EDFA reservoir model [28], an added complexity in SOAs with respect to EDFAs is that scattering losses cannot be neglected. This increases the difficulty in developing a reservoir model for SOAs, and we proposed innovative solutions to tackle the problem. A critical step in the SOA reservoir model is the appropriate selection of the values of its wavelength-dependent parameters that provide a good fit with the experiments. We proposed and described at length a procedure to extract such parameters from the parameters of a detailed and accurate space-resolved SOA model due to Connelly, which we extended to cope with the time-resolved gain transient analysis. It is important to note that our reservoir model is not entirely dependent on the spaceresolved simulator. The key wavelength-dependent parameter for the reservoir model is the material gain as a function of both wavelength and inversion. A detailed knowledge of this dependence allows accurate linearization around the working point and hence, more accuracy for the reservoir model. A procedure to extract the model parameters directly from the measurements would be of great practical value.

A number of other issues remain to be explored and deserve further research. The presence of nonzero facet reflectivity was not considered and would be important for modeling reflective SOAs with the reservoir. In addition, a different approximation for the recombination rate, accounting for a reservoir-dependent time constant, could increase the reliability of the model. In this paper, we assumed a linear dependence of this parameter on the inversion. A better approximation $\left(R(r)=a_{1}(r)+a_{2} r^{2}+\right.$ $\left.a_{3} r^{3}+\ldots\right)$ could be obtained if we assume a constant inversion $N=r / V$ over the SOA length (as we did for ASE calculations in Section III-B). However, the accuracy obtained with such approximations will be at the cost of slower execution time.

The raison d'être of the reservoir model is to find a tradeoff between accuracy and calculation speed. To achieve this goal, we considered several variations of the model, with increasing complexity, which allow the accurate inclusion of both scattering losses and gain saturation induced by ASE. To speed up the emulation of transmission of long bit sequences in the reservoir model, we introduced a single equivalent input ASE channel with appropriate power and gain parameters, which feeds a noiseless reservoir model to give equivalent dynamics. We showed that at a comparable accuracy, the reservoir model with the single ASE channel can be 20 times faster than the Connelly model in single-channel operation, and much more significant time savings are expected for WDM operation. The accuracy of the model is limited to modulation rates per channel not exceeding $10 \mathrm{~Gb} / \mathrm{s}$ since ultrafast phenomena such as $\mathrm{CH}$ and SHB are neglected. However, such rates are of interest for next-generation metropolitan optical networks. In addition, beating-induced carrier gratings that generate FWM and XPM in SOAs are not captured by the reservoir model, which then is reliable whenever XGM dominates over such effects. The true value of the SOA reservoir model is that together with block diagram descriptions of EDFA and Raman amplifiers, it provides a unique tool with reasonably short computation times 
for reliable analysis of gain transients in WDM optical networks with complex topologies.

\section{APPENDIX \\ RESERVOIR EQUATION EXTENDED TO SCATTERING LOSSES}

In this Appendix, we take into account the scattering loss term that was neglected in (13) in the derivation of the reservoir dynamic (14) in order to assess the approximation that we introduced. If one assumes the carrier density along the SOA equals its average value $\bar{N}=r / V(16)$, then

$$
Q_{k}(z) \cong Q_{k}^{\text {in }} e^{\left(\Gamma g_{k}(\bar{N})-\alpha(\bar{N})\right) z}
$$

and the term neglected in (13) can be approximated as

$$
\int_{0}^{L} \alpha(N) Q_{k} d z \cong \alpha(\bar{N}) Q_{k}^{\text {in }} \frac{e^{\left(\Gamma g_{k}(\bar{N})-\alpha(\bar{N})\right) L}-1}{\Gamma g_{k}(\bar{N})-\alpha(\bar{N})}
$$

Since $\left(\Gamma g_{k}(\bar{N})-\alpha(\bar{N})\right) L=a_{k}\left(r-r_{0 k}\right)$, we obtain the "extended reservoir equation with scattering losses" as

$$
\begin{aligned}
\frac{d r(t)}{d t}=\frac{I}{q}-\frac{r(t)}{\tau}-\sum_{k=1}^{n_{\mathrm{sig}}} Q_{k}^{\mathrm{in}} & \left(e^{a_{k}\left(r(t)-r_{0 k}\right)}-1\right) \\
& \times\left(1+\frac{\alpha\left(\frac{r}{V}\right)}{\Gamma g_{k}\left(\frac{r}{V}\right)-\alpha\left(\frac{r}{V}\right)}\right) .
\end{aligned}
$$

Since we can write

$$
1+\frac{\alpha(\bar{N})}{\Gamma g_{k}(\bar{N})-\alpha(\bar{N})}=\frac{g_{k}(\bar{N})}{g_{k}^{\text {net }}(\bar{N})}
$$

it is now evident that the reservoir (14) is accurate whenever $g_{k}^{\text {net }}(\bar{N}) \cong g_{k}(\bar{N})$. However, this is not always guaranteed to hold, especially for deeply saturated SOAs, as one can see for instance in Fig. 7. While in principle one can solve the preceding extended reservoir equation, in this paper, we found it easier to solve the standard reservoir (14) and look for the value of the fluorescence time $\tau$ that gave the best fit to both static and dynamic measurements. In essence, a slightly "decreased" value of $\tau$ has the effect of extra depletion of the reservoir, which has the same qualitative effect of the extra depletion caused by the neglected scattering loss term. Although it is clear that the carrier lifetime has a conceptually different impact on steady-state and transient responses, we chose, by numerical optimization, the value of $\tau$ that leads to the best fit of the measured gain spectrum to that predicted by the steady-state Connelly model. We also show that it is possible to achieve an accurate estimation of the step response at the same time.

\section{ACKNOWLEDGMENT}

A. Bononi would like to thank Dr. Y. Sun for the early discussions about the existence of a reservoir model for SOAs.

\section{REFERENCES}

[1] T. Durhuus, B. Mikkelsen, C. Joergensen, S. L. Danielsen, and K. E. Stubkjaer, "All-optical wavelength conversion by semiconductor optical amplifiers," J. Lightw. Technol., vol. 14, no. 6, pp. 942-954, Jun. 1996.

[2] K. Obermann, S. Kindt, D. Breuer, and K. Petermann, "Performance analysis of wavelength converters based on cross-gain modulation in semiconductor optical amplifiers," J. Lightw. Technol., vol. 16, no. 1, pp. 78-85, Jan. 1998.

[3] D. Marcenac and A. Mecozzi, "Switches and frequency converters based on cross-gain modulation in semiconductor laser amplifiers," IEEE Photon. Technol. Lett., vol. 9, no. 6, pp. 749-751, Jun. 1997.

[4] F. Öhman, S. Bishoff, B. Tromborg, and J. Mørk, "Noise and regeneration in semiconductor waveguides with saturable gain and absorption," IEEE J. Quantum Electron., vol. 40, no. 3, pp. 245-255, Mar. 2004.

[5] M. Zhao, G. Morthier, and R. Baets, "Analysis and optimization of intensity noise reduction in spectrum-sliced WDM systems using a saturated semiconductor optical amplifier," IEEE Photon. Technol. Lett., vol. 14, no. 3, pp. 390-392, Mar. 2002.

[6] M. Menif, W. Mathlouthi, P. Lemieux, L. A. Rusch, and M. Roy, "Errorfree transmission for incoherent broadband optical communications systems using incoherent-to-coherent wavelength conversion," J. Lightw. Technol., vol. 23, no. 1, pp. 287-294, Jan. 2005.

[7] A. Uskov, J. Mork, and J. Mark, "Theory of short-pulse gain saturation in semiconductor laser amplifiers," IEEE Photon. Technol. Lett., vol. 4, no. 5, pp. 443-446, May 1992.

[8] J. Mork and A. Mecozzi, "Theory of the ultrafast response of active semiconductor waveguides," J. Opt. Soc. Amer. B, Opt. Phys., vol. 13, no. 8, pp. 1803-1816, Aug. 1996.

[9] A. Mecozzi, S. Scotti, A. D’Ottavi, E. Iannone, and P. Spano, "Four-wave mixing in traveling-wave semiconductor amplifiers," IEEE J. Quantum Electron., vol. 31, no. 4, pp. 689-699, Apr. 1995.

[10] D. Marcuse, "Computer model of an injection laser amplifier," IEEE J. Quantum Electron., vol. QE-19, no. 1, pp. 63-73, Jan. 1983.

[11] T. Durhuus, B. Mikkelsen, and K. E. Stubkjaer, "Detailed dynamic model for semiconductor optical amplifiers and their crosstalk and intermodulation distortion," J. Lightw. Technol., vol. 10, no. 8, pp. 1056-1065, Aug. 1992.

[12] M. J. Connelly, "Wideband semiconductor optical amplifier steadystate numerical model," IEEE J. Quantum Electron., vol. 37, no. 3, pp. 439-447, Mar. 2001.

[13] G. P. Agrawal and N. A. Olsson, "Self phase modulation and spectral broadening of optical pulses in semiconductor laser amplifier," IEEE J. Quantum Electron., vol. 25, no. 11, pp. 2297-2306, Nov. 1989.

[14] A. A. M. Saleh, "Non linear models of travelling wave optical amplifiers," Electron. Lett., vol. 24, no. 14, pp. 835-837, Jul. 1988.

[15] A. A. M. Saleh and I. M. I. Habbab, "Effects of semiconductor optical amplifier nonlinearity on the performance of high-speed intensitymodulation lightwave systems," IEEE Trans. Commun., vol. 38, no. 6, pp. 839-846, Jun. 1990.

[16] C. Tai and W. I. Way, "Dynamic range and switching speed limitations of an $N \times N$ optical packet switch based on low-gain semiconductor optical amplifiers," J. Lightw. Technol., vol. 14, no. 4, pp. 962-967, Apr. 1992.

[17] T. Liu, K. Obermann, K. Petermann, F. Girardin, and G. Guekos, "Effect of saturation caused by amplified spontaneous emission on semiconductor optical amplifier performance," Electron. Lett., vol. 33, no. 24, pp. 2042-2043, Nov. 1997.

[18] G. Talli and M. J. Adams, "Gain dynamics of semiconductor optical amplifiers and three-wavelength devices," IEEE J. Quantum Electron., vol. 39, no. 10, pp. 1305-1313, Oct. 2003.

[19] M. M. Freire and H. J. A da Silva, "Performance implications of partial chirp compensation in a semiconductor optical booster amplifier for dispersion supported transmission at $10 \mathrm{~Gb} / \mathrm{s}$," in Proc. ICT, Jun. 1998, vol. 1, pp. 76-80.

[20] M. Settembre, F. Matera, V. Hägele, I. Gabitov, A. W. Mattheus, and S. K. Turitsyn, "Cascaded optical communication systems with in-line semiconductor optical amplifier," J. Lightw. Technol., vol. 15, no. 6, pp. 962-967, Jun. 1997.

[21] A. Bononi and L. A. Rusch, "Doped-fiber amplifier dynamics: A system perspective," J. Lightw. Technol., vol. 16, no. 5, pp. 945-956, May 1998. 
[22] Y. Sun, J. L. Zyskind, and A. K. Srivastava, "Average inversion level, modeling, and physics of erbium-doped fiber amplifiers," IEEE J. Sel. Topics Quantum Electron., vol. 3, no. 4, pp. 991-1007, Aug. 1997.

[23] R. Ramaswami and P. A. Humblet, "Amplifier induced crosstalk in multichannel optical networks," J. Lightw. Technol., vol. 8, no. 12, pp. 1882-1896, Dec. 1990.

[24] E. Tangdiongga, J. J. J. Crijns, L. H. Spiekman, G. N. Van den Hoven, and H. de Waardt, "Performance analysis of linear optical amplifiers in dynamic WDM systems," IEEE Photon. Technol. Lett., vol. 14, no. 8, pp. 1196-1198, Aug. 2002.

[25] S. Novak and A. Moesle, "Simulink model for EDFA dynamics applied to gain modulation," J. Lightw. Technol., vol. 20, no. 6, pp. 986-992, Jun. 2002.

[26] A. Bononi, M. Papararo, and M. Fuochi, "Transient gain dynamics in saturated Raman amplifiers," Opt. Fiber Technol., vol. 10, no. 1, pp. 91-123, Jan. 2004.

[27] E. Kreyszig, Advanced Engineering Mathematics, 7th ed. New York: Wiley, 1993

[28] L. Tancevski, A. Bononi, and L. A. Rusch, "Output power and SNR swings in cascades of EDFAs for circuit- and packet-switched optical networks," J. Lightw. Technol., vol. 17, no. 5, pp. 733-742, May 1999.

[29] A. A. Rieznik and H. L. Fragnito, "Analytical solution for the dynamic behavior of erbium-doped fiber amplifiers with constant population inversion along the fiber," J. Opt. Soc. Amer. B, Opt. Phys., vol. 21, no. 10, pp. 1732-1739, Oct. 2004.

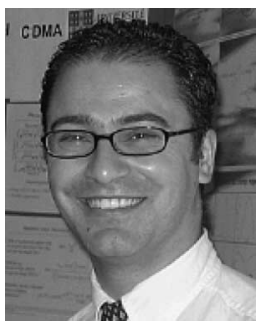

Walid Mathlouthi received the B.S.E.E. degree from École Nationale d'Ingénieurs de Tunis, Tunis, Tunisia, and the M.S.E.E degree from Université Laval, Québec City, QC, Canada. He is currently working toward the Ph.D. degree in electrical engineering at the Center of Optics, Photonics and Lasers, Département de Génie Électrique et Informatique, Université Laval.

His research interests include semiconductor optical amplifiers, spectrum-sliced wavelength-division multiplexing, fiber Bragg gratings, optical codedivision multiple access, and wireless optical communications.

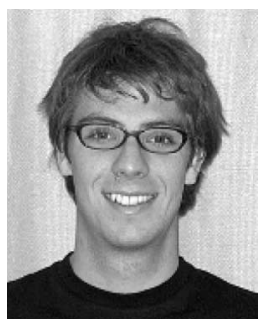

Pascal Lemieux received the B.S. degree (with honors) in engineering physics from Université Laval, Québec City, QC, Canada, in 2004. He is currently working toward the M.S. degree in optical telecommunications at the Centre d'Optique, Photonique et Laser, Département de Génie Électrique et Informatique, Université Laval.

His research interests include semiconductor optical amplifiers, spectrum-sliced wavelength-division multiplexing, statistical properties of optical light sources, optical code-division multiple access, and wireless optical communications.

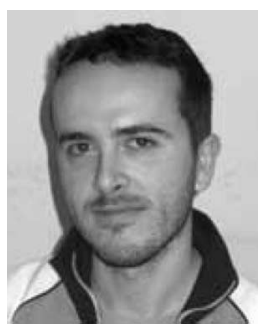

Massimiliano Salsi was born in Parma, Italy, in 1979. He received the Laurea degree (cum laude) in telecommunications engineering from the University of Parma, Parma, in 2004. He is currently working toward the Ph.D. degree in information technology at the Department of Information Engineering, University of Parma.

His current research interests include saturation, stabilization, and nonlinear effects in optical amplifiers for wavelength-division-multiplexing long-haul and metropolitan-networks applications.

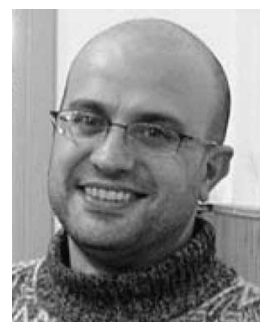

Armando Vannucci ( $\left.\mathrm{S}^{\prime} 95-\mathrm{M}^{\prime} 01\right)$ was born in Frosinone, Italy, in 1968. He received the Laurea degree (cum laude) in electronics engineering from the University of Roma "La Sapienza," Rome, Italy, and the Ph.D. degree in information engineering from the University of Parma, Parma, Italy, in 1993 and 1998, respectively.

Until 1995, he was with the INFO-COM Department, University of Roma. Since 1995, he has been with the Department of Information Engineering, University of Parma, where he is currently an Assistant Professor of telecommunications. His current research interests include optical communication systems and polarization mode dispersion, nonlinear wavelength-division-multiplexing fiber transmission, system performance evaluation, and optical amplifiers.

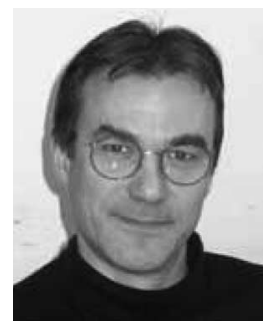

Alberto Bononi received the Laurea degree (cum laude) in electronics engineering from the University of Pisa, Pisa, Italy, in 1988 and the M.A. and $\mathrm{Ph} . \mathrm{D}$. degrees in electrical engineering from Princeton University, Princeton, NJ, in 1992, and 1994, respectively.

He is currently an Associate Professor of telecommunications with the Department of Information Engineering, School of Engineering, University of Parma, Parma, Italy, where he teaches courses in probability theory and stochastic processes, telecommunications networks, and optical communications. In 1990, he was with GEC-Marconi Hirst Research Centre, Wembley, U.K., where he worked on a Marconi S.p.A. project on coherent optical systems. From 1994 to 1996, he was an Assistant Professor with the Department of Electrical and Computer Engineering, State University of New York, Buffalo, where he taught courses in electric circuits and optical networks. In summers 1997 and 1999, he was a Visiting Faculty with the Département de Genie Électrique, Université Laval, QC, Canada, where he did research on fiber amplifiers. His current research interests include system design and performance analysis of high-speed all-optical networks, nonlinear fiber transmission for wavelength-division-multiplexing systems, linear and nonlinear polarization mode dispersion, and transient gain dynamics in optical amplifiers.

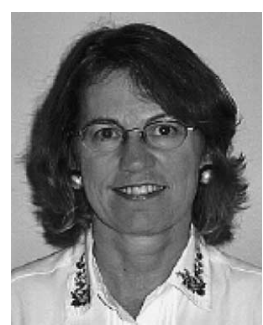

Leslie A. Rusch (S'91-M'94-SM'00) received the B.S.E.E. degree (with honors) from California Institute of Technology, Pasadena, in 1980 and the M.A. and Ph.D. degrees in electrical engineering from Princeton University, Princeton, NJ, in 1992 and 1994 , respectively.

From 2001 to 2002, she was with Intel Corporation as the Manager of a group that researches new wireless technologies. She is currently a Full Professor with the Department of Electrical and Computer Engineering, Université Laval, Québec City, QC, Canada, performing research in wireless and optical communications. Her research interests include optical code-division multiple access using noncoherent sources for metropolitan area networks, semiconductor and erbiumdoped optical amplifiers and their dynamics, and wireless-communications high-performance reduced-complexity receivers for ultrawideband systems employing code-division multiple access. 